\title{
Kosten und Nutzen kleiner Spitäler
}

\author{
Hans Hoppeler ${ }^{a, b}$ \\ Oliver Richner ${ }^{b}$, \\ Markus Bieric, \\ Jürg Müller ${ }^{b}$

\section{b MKR, Solothurn \\ c Ärztenetzwerk Oberes Emmental, Langnau} \\ a Institut für Anatomie, Bern
}

Die in diesem Artikel wiedergegebenen Äusserungen repräsentieren die Meinung der Autoren in dieser Sache und nicht jene der Universität Bern.

Korrespondenz:

Prof. Dr. med. H. Hoppeler Universität Bern Institut für Anatomie Baltzerstrasse 2 CH-3000 Bern

Tel. 0316314637

hoppeler[at]ana.unibe.ch

\section{Hintergrund}

Das in Diskussion stehende Spitalversorgungsgesetz des Kantons Bern hält fest, dass im Kanton Bern eine Spitalüberkapazität besteht, welche weiter abgebaut werden sollte. In der Vergangenheit hat die Sorge um diese Überkapazität hauptsächlich dazu geführt, dass viele der kleineren Akutspitäler in der Peripherie aufgrund ihrer ungünstigen Kostenstruktur geschlossen wurden. Die vorliegende Untersuchung setzt sich kritisch mit den wirtschaftlichen Schliessungskriterien für Kleinspitäler auseinander.

Am publizierten Zahlenmaterial für die Schweiz gibt es wenig Hinweise dafür, dass grössere Spitäler immer günstiger arbeiten. Betrachtet man Fallkosten und Fallzahlen (Spital-Benchmark, Daten 2006) unvoreingenommen, so wird deutlich, dass Spitäler mit Fallzahlen zwischen 3000 und 15000 die niedrigsten Fallkosten aufweisen, auch wenn dieser Bericht die günstigste Spitalgrösse bei 10000-12000 ansiedelt. Die Untersuchung zeigt aber auch, dass die Fallkosten ab ca. 20000 Fällen pro Jahr und Spital ansteigen. Es ist zu vermuten, dass dieser Anstieg auf die steigende Komplexität grosser Spitalgebilde und der damit verbundenen Ineffizienzen (zusätzliche Hierarchiestufen, Kommunikationskosten, Sortiment, Technologieansprüche usw.) zurückzuführen ist. Ähnliche Daten aus der Spital-Benchmark 2011 zeigen, dass Spitäler zwischen etwa 80 und 300 Betten am günstigsten operieren, allerdings mit einer spitalgrössenunabhängigen, breiten Streuung.

Im Kanton Bern finden wir kleinere Spitäler häufig in einem Spitalverbund mit einem grösseren Mutterhaus. Dabei stehen stets die kleineren Spitäler unter Schliessungsdruck. Aus Spargründen wurden in kleineren Spitälern in den letzten Jahren immer wieder Leistungen abgebaut und auf Investitionen verzichtet. Dies hat in der Bevölkerung, aber auch unter den Hausärzten der betroffenen Spitalregionen Unbehagen ausgelöst, so zum Beispiel in Langnau. Das Spital Langnau ist der «kleinere Partner» des Regionalspitals Emmental (RS-E). Das Spital Langnau (2616 Fälle stationär, 8078 ambulant für 2011) bildet zusammen mit dem Spital Burgdorf (5953 Fälle stationär, 30055 ambulant) das Regionalspital Emmental, das für diesen Teil des Kantons vom Regierungsrat den Leistungsauftrag für die Versorgung der Bevölkerung hat.

Um die Situation des Spitals Langnau darzustellen, werden im vorliegenden Bericht Kosten und Erträge verursachergerecht auf die Standorte Burgdorf und Langnau verteilt (Ist-Zustand). Dazu wurde ein rechnungslegungsunabhängiges parametrisiertes

\section{Rapport coût-efficacité des petits hôpitaux}

Depuis près de vingt ans, de petits hôpitaux sont fermés pour cause de structures de coûts défavorables. Selon des données indiquées par «SpitalBenchmark», les hôpitaux traitant 3000 à 15000 cas par année ont, avec une grande dispersion, les structures de coûts les plus avantageuses. Les petits hôpitaux souffrent de frais fixes élevés, tandis que les grands hôpitaux doivent affronter des frais de complexité. Un modèle paramétré a été développé pour le Regionalspital Emmental (Burgdorf 154 lits / 6000 cas et Langnau 66 lits / 2600 cas). Celui-ci permet une allocation des coûts et produits pour chaque site en fonction de la source et indépendamment de la comptabilité. Ce modèle démontre que l'hôpital de Langnau est, actuellement, juste au-dessus du point breakeven. La modélisation de différents scénarios, comme la fermeture de Langnau sans aucune compensation ou la transformation de Langnau en centre de santé, nuit à la profitabilité du groupe Regionalspital Emmental. Cela est surtout dû aux investissements et amortissements extraordinaires causés par la fermeture et la délocalisation. De plus, le modèle calcule les coûts d'économie générale provoqués par les transports supplémentaires de patients et visiteurs ainsi que les coûts de communication des médecins de famille et les incidences sur la fiscalité régionale. Le modèle relève aussi les effets positifs d'un agrandissement modéré de cet hôpital et, de ce fait, la consolidation de la situation de I'hôpital en tant que base d'un approvisionnement durable de la région en médecins de famille.

Modell erstellt mit dem Ziel, mögliche Zukunftsszenarien für das RS-E abzubilden, nämlich:

- ersatzlose Schliessung des Spitals Langnau,

- Schliessung des Spitals Langnau mit Betrieb eines Gesundheitszentrums,

- Ausbau des Spitals Langnau. 
Als wichtiges Element werden im vorliegenden Modell auch volkswirtschaftliche Kosten wie z.B. zusätzliche Patiententransporte, Kosten für Besuchertransporte sowie lokale Steuerausfälle ausgewiesen. Der Einbezug einer gemeinwirtschaftlichen Sichtweise scheint uns dabei wesentlich; für den Steuerzahler sind letztlich nicht die Spitalbudgets relevant, sondern die gesamthaft anfallenden Gesundheitskosten.

\section{Vorgehen}

Im Rahmen der Datenerhebung wurden semistrukturierte Interviews von jeweils circa einer Stunde mit den Hausärzten (4), der Spitalleitung (4), dem Pflegedienst (3) und den Spitalärzten (2) geführt.

Die finanziellen Parameter wurden aufgrund der Erfolgsrechnung des RS-E ermittelt, die uns von der Verwaltung des RS-E zur Verfügung gestellt wurde. Da das RS-E keine standortspezifische Rechnung führt, eignet sich die Erfolgsrechnung des RS-E nicht für eine Abklärung mit Modellcharakter. Wir mussten deshalb die Modellrechnungen auf eine vom Rechnungswesen unabhängige Basis stellen. Dies mit dem Vorteil, dass das parametrisierte Modell auch auf andere Spitäler oder Spitalverbände anwendbar ist.

Die Positionen der Aufwand- und der Ertragsseite der Erfolgsrechnung RS-E wurden für die Parametrisierung einer von vier Kategorien wie folgt zugeteilt:

a) Positionen, die sich zum Personaleinsatz proportional verhalten.

b) Positionen, die sich zu den Fallzahlen proportional verhalten, wie zum Beispiel Arzneimittel, Material usw.

c) Positionen, die sich zur Spitalgrösse (Bettenzahl) proportional verhalten, wie z. B. Unterhalt, Energieverbrauch usw.

d) Positionen, die sich zur Hospitalisationsdauer proportional verhalten, z. B. die Hotelleriekosten.

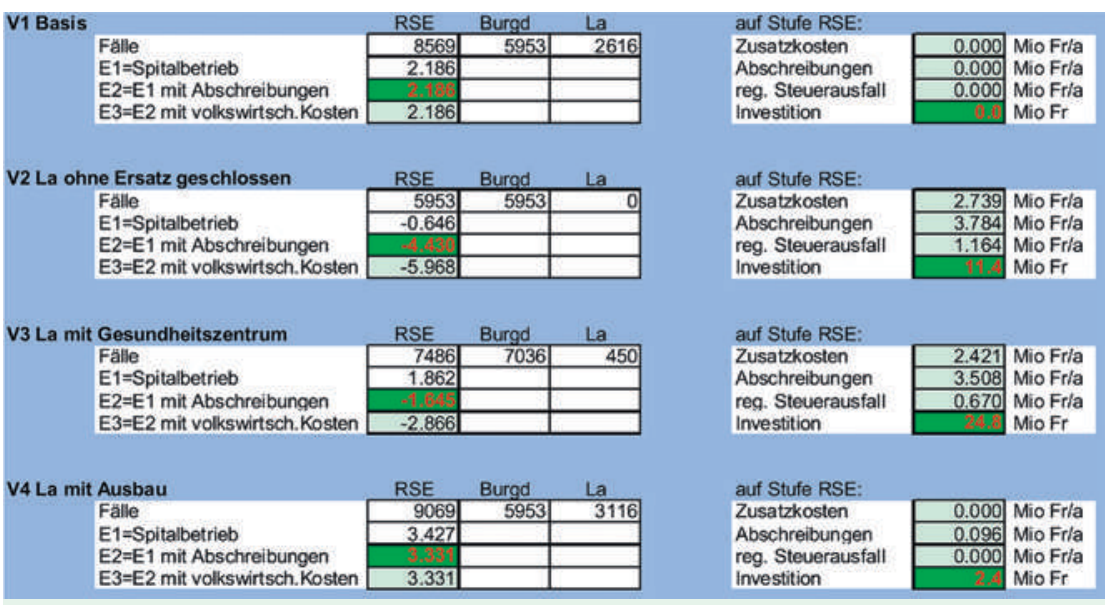

Tabelle 1

Ertragsberechnung für die vier gerechneten Varianten

E1 = Ertrag Spitalbetrieb allein, E2 = Ertrag Spitalbetrieb nach Abzug der Abschreibungen und E3 = Ertrag Spitalbetrieb nach Abzug der Abschreibungen und der volkswirtschaftlichen Kosten Die Zusatzkosten umfassen die Kosten für den Betrieb zusätzlicher Rettungsteams, Verlagerungskosten Personal, zusätzliche Kosten der Hausärzte sowie zusätzliche Transportkosten.
Jeder Position in den vier oben definierten Kategorien wurde zusätzlich ein Fixkostenanteil zugeordnet. Dabei wurde berücksichtigt, welchen Kostenanteil eine einzelne Position in einem voll funktionsfähigen Spital ohne Patienten verursachen würde. Die aus verschiedenen Regularien erforderlichen Vorhalteleistungen oder Randbedingungen aus dem Gesamtarbeitsvertrag gingen dabei ebenfalls in die Fixkostenanteile ein. Diese Vorgehensweise gestattet es, wiederkehrende Kosten vom Rechnungswesen unabhängig in einer Modellrechnung darzustellen.

Bei einer Spitalschliessung fallen zusätzlich einmalige Kosten an. Diese haben den Charakter von Investitionen und müssen abgeschrieben werden. Je nach Variante müssen Betten verlagert werden und Bettenkapazitäten aufgebaut respektive stillgelegt werden. Ebenfalls müssen die Integrations-, Rekrutierungs- und Entlassungskosten für das Personal berücksichtigt werden. Für die Verlagerung eines Bettes haben wir einen Investitionsbedarf von $200000 \mathrm{CHF}$, eine Abschreibung über 15 Jahre; für eine Kapazitätserweiterung um ein Bett einen Investitionsbedarf von $800000 \mathrm{CHF}$, eine Abschreibung über 25 Jahre angenommen. Die Kosten von Integration, Rekrutierung und Entlassung von Personal haben wir mit 8000 CHF, 6000 CHF resp. 30000 CHF pro Person eingesetzt bei einer Abschreibungsdauer von drei Jahren. Stilllegungskosten haben wir mit 100000 CHF pro Bett beziffert mit einer Abschreibungsdauer von drei Jahren.

Aus den wiederkehrenden und den einmaligen Kosten lassen sich die effektiven Kosten in der Betriebsrechnung für die unterschiedlichen Modellvarianten ermitteln. Diese Daten sind für die Spitalrechnung relevant, berücksichtigen aber noch nicht alle Kosten, die durch Spitalverlagerungen ausgelösten werden. Wir haben diese Kosten als volkswirtschaftliche Kosten bezeichnet.

$\mathrm{Zu}$ den volkswirtschaftlichen Kosten haben wir den zusätzlichen Betrieb eines Rettungsteams zur Erfüllung der kantonalen Rettungsvorgaben gerechnet (Betrag 1200000 CHF), Mehrkosten der Hausärzte für Kommunikation mit einem weiter entfernten Spital (590000 CHF), Mehrkosten durch die längeren Fahrstrecken der Besucher (630000 CHF) und der ambulanten Patienten (320000 CHF). Diese volkswirtschaftlichen Kosten erscheinen nicht in der Spitalrechnung, sondern werden den Patienten, den Familien der Patienten oder den Hausärzten aufgebürdet und werden damit letztlich auch vom Steuerzahler beglichen.

Von lokalpolitischer Bedeutung sind auch Steuerausfälle, die mit einer Spitalschliessung einhergehen. Diese umfassen Ausfälle von Einkommenssteuer bei Wegzug oder bei Nichtweiterbeschäftigung von Personal sowie Steuerausfälle, verursacht durch den Wegfall des Spitals als Leistungsbezieher für das lokale Gewerbe (zusammen 1164000). 


\section{Quantitative Ergebnisse und Diskussion der Modellvarianten}

Wie bereits eingangs erwähnt, handelt es sich bei den errechneten Beträgen für die verschiedenen Modellvarianten um Schätzungen, ausgehend von einem Ist-Zustand (Modell-Variante 1; Tab. 1). Es handelt sich also im Wesentlichen um einen von der Grundsituation ausgehenden Vergleich der Varianten. Dies bedeutet, dass - auch wenn einzelne Absolutwerte nicht korrekt sein sollten - trotzdem Vergleichbarkeit zwischen den Varianten besteht. Insbesondere lassen sich sensitive und weniger sensitive Parameter identifizieren.

Modell-Variante 1 - Ist-Zustand: Die Standorte Burgdorf und Langnau im Regionalspital Emmental erbringen sehr unterschiedliche Leistungen. Während Burgdorf (154 Betten) eine breite Palette medizinischer Leistungen anbietet (inklusive Geburtshilfe und einer Intensivpflege), konzentriert sich Langnau (66 Betten) vor allem auf die Gebiete Innere Medizin, Chirurgie/Orthopädie, Gynäkologie und Komplementärmedizin im Rahmen einer erweiterten, spitalbasierten Grundversorgung. Es gibt keine permanent in Langnau stationierte Anästhesiologie. Im operativen Bereich betreibt Langnau vor allem elektive Chirurgie, Orthopädie und Gynäkologie, wobei umfangreichere Eingriffe in der Regel in Burgdorf vorgenommen werden. Aufwand und Ertrag für Burgdorf und Langnau sind fallzahlenabhängig in Abbildung 1 dargestellt. Der Gesamterfolg des RS-E beträgt
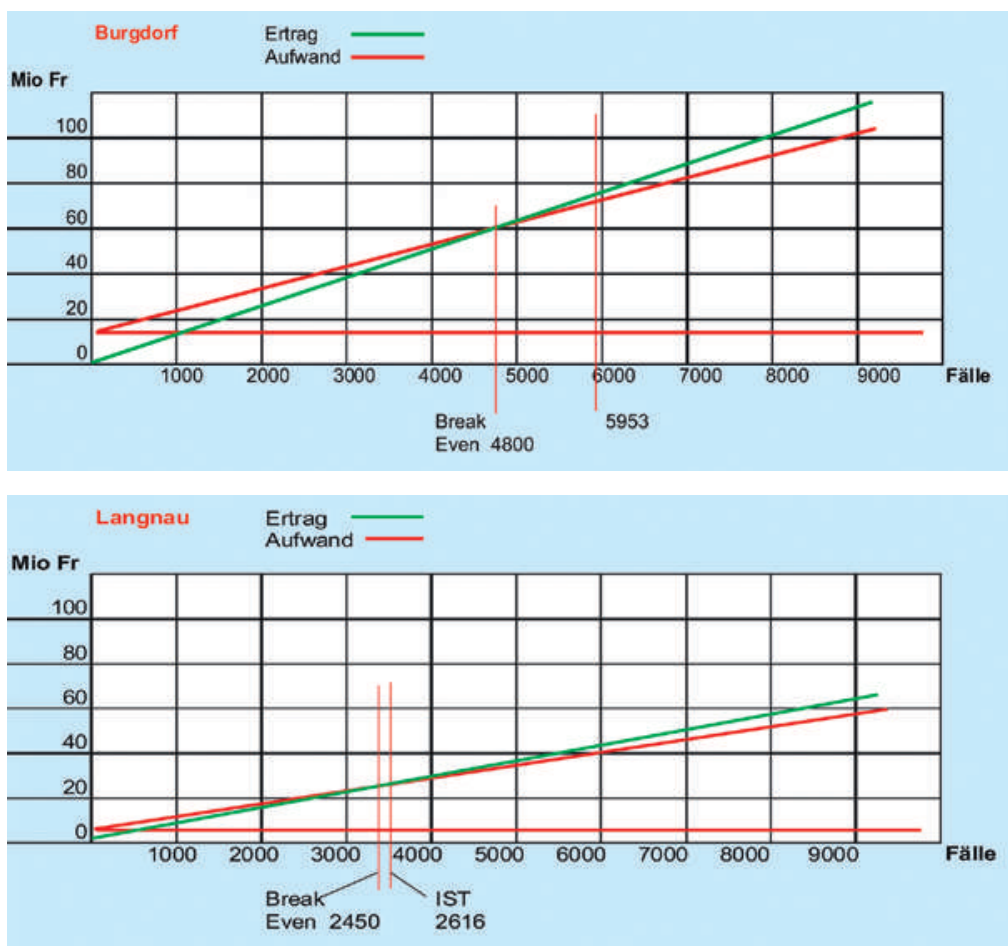

Abbildungen $1 \mathrm{a}$ und $\mathbf{1 b}$

Ertrag und Aufwand der Spitäler Burgdorf und Langnau in Funktion der Fallzahlen. Beim Aufwand ist der Fixkostenanteil (horizontale Linie) vom variablen Aufwand (ansteigende Linie) unterschieden.
2186000 CHF. Nominal fallen davon in Burgdorf 1554000 CHF, in Langnau 632000 CHF an. Der Erfolg in Langnau muss allerdings um etwa 400000 CHF nach unten korrigiert werden, weil die Kosten für verschiedene in Langnau tätige Operateure in Burgdorf anfallen.

Modell-Variante 2 - Ersatzlose Schliessung von Langnau: In dieser Variante wird angenommen, dass alle Patienten von anderen Anbietern aufgenommen würden. Diese Annahme lässt sich dadurch rechtfertigen dass ja kantonsweit ein Überangebot an Spitalbetten besteht. In dieser Variante fallen damit in Langnau «nur» Schliessungskosten in der Höhe von $6600000 \mathrm{CHF}$ an sowie volkswirtschaftliche Kosten und Steuerausfälle. Zusätzlich vermindert sich der Ertrag des RS-E durch den Ausfall der Langnauer Fälle. Es muss zudem berücksichtigt werden, dass zur Sicherstellung der medizinischen Versorgung in Langnau ein zusätzliches Rettungsteam stationiert werden sollte (12000000 CHF, Tab. 1).

Modell-Variante 3 - Schliessung des Spitals, mit Aufbau eines Gesundheitszentrums: Bei dieser Variante wurde angenommen, dass in den vorhandenen Spitalstrukturen ein Gesundheitszentrum aufgebaut würde. Neben Privatpraxen würden weiterhin maximal 10 Tagesbetten zur Verfügung stehen. Wir sind davon ausgegangen, dass weiterhin 50\% der Patienten im Spital Burgdorf behandelt würden. Es müssten also in Langnau Betten abgebaut und in Burgdorf aufgebaut werden. Für einen Teil des Personals würden Kosten für Integration, Rekrutierung und Entlassung anfallen. Weiterhin würden volkswirtschaftliche Kosten durch zusätzliche Kosten der Hausärzte (589000 CHF), zusätzliche Transporte der Besucher (632000 CHF), und durch den Einsatz eines zusätzlichen Rettungsteams (1200000 CHF) anfallen. Es würde zu einem Steuerausfall durch Minderbeschäftigung in der Region und durch den Wegfall der Bezüge des Spitals bei den lokalen Lieferanten (670000 CHF) kommen.

Modell-Variante 4 - Ausbau des Spitals Langnau: Wie bereits im Ist-Zustand festgehalten, bewegt sich das Spital Langnau nahe am Break-even-Punkt. Es müsste deswegen versucht werden, die Fallzahlen in Langnau zu erhöhen. Die zuweisenden Ärzte beklagen vor allem den Mangel eines permanent am Spital eingesetzten Orthopäden. Eine Reakquisition von Fällen aus dem benachbarten oberen Entlebuch erscheint möglich, da unter DRG keine Kantonsgrenzen für grundversicherte Patienten mehr bestehen. Für diese Modellvariante haben wir mit einer Fallzahl von zusätzlichen 500 Fällen gerechnet. In dieser Variante würde der Ertrag Langnau von 200000 CHF auf 1873000 CHF angehoben, was den Gesamtertrag des RS-E auf 3331000 CHF anheben würde.

In allgemeiner Form sind die Resultate der Modellrechnung in Abbildung 2a und 2b festgehalten. Es zeigt sich dabei, dass sich, unter Berücksichtigung 


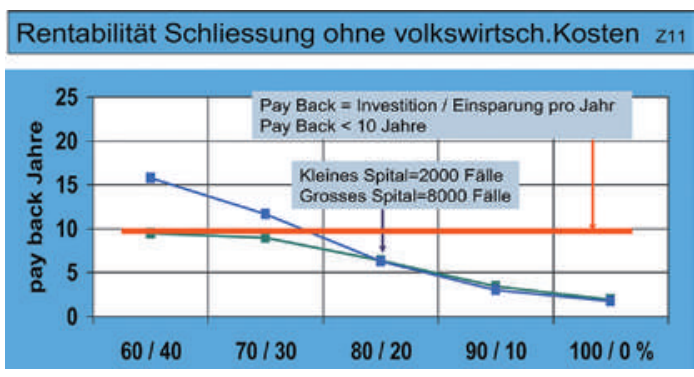

Rentabilität Schliessung mit volkswirtsch.Kosten $\quad$ z10

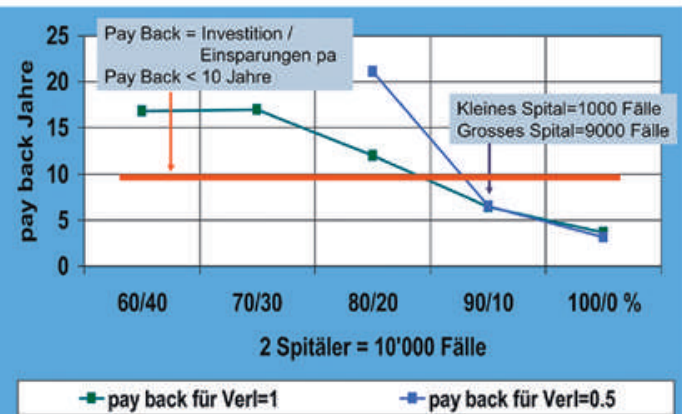

Abbildungen $2 \mathrm{a}$ und $2 \mathrm{~b}$

Modellrechnung ausgehend von zwei Spitälern mit einer Gesamtfallzahl von 10000 . Werden, bei Schliessung des kleineren Spitals, die volkswirtschaftlichen Kosten nicht berücksichtigt, kann ein vernünftiger Payback von 10 Jahren erwartet werden, wenn die Fallzahlen des kleineren Spitals $<4000$ sind. Dies allerdings nur, wenn $100 \%$ der Patienten vom grösseren Spital aufgenommen werden. Erhält das verbleibende Spital nur 50\% der Fälle, liegt eine rentable Schliessungsgrenze bei etwa 2500 Fällen für das kleinere Spital. Werden die volkswirtschaftlichen Kosten mitgerechnet, verschiebt sich eine rentable Schliessungsgrenze in den Bereich von etwa 1000 Fällen für das kleinere Spital.

der volkswirtschaftlichen Kosten, eine Spitalschliessung eines Spitals mit 2000 Fällen wohl in den meisten Fällen wirtschaftlich nicht rechtfertigen lässt.

\section{Qualitative Argumente zur Bedeutung peripherer Akutspitäler}

Das periphere Akutspital als Weiterbildungsstätte Wir haben festgestellt, dass die meisten der im Einzugsgebiet des Spitals Langnau tätigen Hausärzte einen Teil ihrer Weiterbildungszeit am Spital Langnau (oder in einem vergleichbaren Kleinspital) geleistet haben. Es wird angegeben, dass dies für die Berufsentscheidung und die Wahl des Praxisstandortes von ausschlaggebender Bedeutung war. Damit kommt einem peripheren Akutspital für die nachhaltige Versorgung der Region mit Hausärzten eine grundlegende Bedeutung zu.

\section{Das periphere Akutspital als medizinische Ressource}

Im Zusammenhang mit der Fort- und Weiterbildungstätigkeit an einem peripheren Akutspital entsteht ein enges lokales Netzwerk, bestehend aus Spital- und Hausärzten. Dies bringt in der Kommunika- tion eine erhebliche Erleichterung. Die zunehmende Spezialisierung und die kürzeren Weiterbildungszeiten formen einen neuen Typ von Grundversorgern. Die kommende Generation von Hausärzten und Hausärztinnen arbeiten nicht mehr allein, sondern im Praxisverbund, exponierte Praxisstandorte werden gemieden. Ein peripheres Akutspital kann die Hausärzte in der Notfallversorgung unterstützen. Bei Verlust dieser Akutspitäler ist damit zu rechnen, dass mittelfristig auch die Hausärzte in der Peripherie fehlen werden.

\section{Bevölkerung und peripheres Akutspital}

Patienten, die im peripheren Akutspital hospitalisiert werden, werden weniger aus ihrem sozialen Kontext herausgerissen. Das Spital ist in der Nähe, Besuchswege sind kurz und viele der (häufig teilzeitarbeitenden) Pflegepersonen stammen aus der Region. Es sind dies qualitative Unterschiede, die sich letztlich positiv auf den Heilungsverlauf und auf die Hospitalisationsdauer auswirken. Der Erhalt peripherer Akutspitäler entspricht auch dem Trend, die Versorgung einer alternden Bevölkerung so peripher wie möglich zu gestalten. Es sei dabei nur am Rande angemerkt, dass jede korrekt indizierte periphere Hospitalisierung kostengünstiger ist, als eine Hospitalisierung in einem zentral gelegenen Spital.

\section{Abschliessende Überlegungen}

Die Gesamtbeurteilung der Situation zeigt, dass Langnau gerade eben noch rentabel operiert. Allerdings liegt der Break-even-Punkt von Langnau nahe an den heutigen Fallzahlen. Es scheint deswegen sinnvoll, Massnahmen zu ergreifen, welche die Fallzahlen in Langnau erhöhen. Die Schliessung von Langnau oder der Betrieb eines Gesundheitszentrums schneiden vor allem wegen der hohen Investitionskosten und den damit verbundenen Abschreibungen schlecht ab. Periphere Akutspitäler haben zusätzlich eine Rolle als «Brutstätte» und wichtigste Anlaufstelle für die lokalen Hausärzte und helfen diesen, die Notfallversorgung sicherzustellen. Sie sind damit Garant für eine nachhaltige Versorgung der Peripherie mit Hausärzten.

\section{Danksagung}

Die vorliegende Untersuchung wurde vom «Ärztenetzwerk Oberes Emmental» ermöglicht. Für die Mitarbeit der Hausund Spitalärzte, der Pflegepersonen und der Verwaltung der untersuchten Institutionen bedanken sich die Autoren.

\section{Referenzen}

- Spital-Benchmark 2006 www.spitalbenchmark.ch/frontend/documents/ public/content/cmVzdWx0cy0xMjE5NjUwODU3.pdf

- Spitalversorgungsgesetz Bern, Version 4. Juli 2012 www.be.ch/portal/de/index/mediencenter/ medienmitteilungen.assetref/content/dam/ documents/portal/Medienmitteilungen/de/2012/ 07/2012-07-05-vortrag_spitalversorgungsgesetz.pdf 